\section{Los caminos de la extensión en la universidad argentina}

Castro, J. y Oyarbide, F. (comps.) (2015).

Santa Rosa: Universidad Nacional de La Pampa.

168 páginas. Disponible en versión digital.

Reseñado por Pablo Arnau Short

Universidad Nacional del Litoral

pablo_arnaushort@hotmail.com
Integración de la docencia y la extensión /

Reseña de libros
Se trata de una compilación que expone de una forma clara el parcours de las últimas décadas de diferentes experiencias extensionistas a partir de la socialización de determinadas prácticas universitarias/comunitarias con el firme propósito de consolidar la integración de las tres funciones sustantivas de la universidad. El aporte general reposa en la consideración de la extensión situada en tiempo-espacio-sujetos. Todo ello es puesto en escena bajo el telón de fondo de la reclamada "curricularización de la extensión". En este sentido, todos los artículos (algunos más, otros menos) ponen énfasis en este proceso.

El Capítulo 1 realiza una reconstrucción histórica y teórica de la extensión a partir de una interesante relectura del nuevo impulso extensionista desde la crisis de 2001 en clave epistemológica. El Capítulo 2 revaloriza y resignifica la función de extensión a través de la instrumentalización de algunos mecanismos específicos tales como la "categoría de docente-extensionista" en la Universidad Nacional de la Patagonia Austral. El Capítulo 3 analiza los dos dispositivos de extensión más importantes con los que cuenta la UNL: el Sistema Integrado de Proyectos y Programas de Extensión (instrumento clave para la planificación y gestión de la extensión universitaria del litoral) y los Centros de Extensión Comunitaria (modelo de intervención micro y macro social en varias comunas y municipios de la provincia de Santa Fe). El Capítulo 4 representa una buena síntesis conceptual acerca de las diferentes concepciones de extensión planteando también aquellos problemas entre discursos progresistas y prácticas conservadoras en torno a la extensión en la Universidad Nacional de Cuyo. El Capítulo 5 pone en valor el rol de la autocrítica dentro del marco de avance extensionista en la Universidad Nacional de Rosario con base en el trabajo realizado en territorios específicos ("villas" y "asentamientos") con la consecuente "construcción social del conocimiento". El Capítulo 6 evalúa dos programas llevados adelante por la Universidad Nacional del Nordeste: uno, denominado UNNE Salud (donde se trabaja con pueblos originarios de las ciudades de Corrientes y Resistencia); otro, llamado la Universidad en el Medio (donde se trabajan aspectos culturales

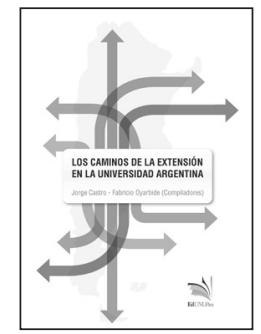

del entorno social con la participación de no-docentes). El Capítulo 7 representa la socialización de las primeras experiencias de curricularización de la extensión en la Universidad Nacional de Entre Ríos a partir de la puesta en valor de las diferentes instancias institucionales que debieron superar. Actualmente, se están evaluando estas primeras prácticas y el Consejo Superior ha lanzado una nueva convocatoria en donde lo más sobresaliente lo constituye la posibilidad de presentación de proyectos de extensión dirigidos por estudiantes avanzados. El Capítulo 8 discute la problemática de hacer visible y acreditable las prácticas de extensión en la Universidad Nacional del Centro de la Provincia de Buenos Aires. El Capítulo 9 problematiza, desde la Universidad Nacional del Noroeste de la Provincia de Buenos Aires, las relaciones entre la extensión universitaria y los conceptos vertidos por la UNESCO acerca del "compromiso social comunitario". El Capítulo 10 pone énfasis en las "prácticas sociocomunitarias" desarrolladas en la Universidad Nacional de Mar del Plata como reclamo surgido por el claustro de estudiantes y como actual forma de articulación entre la extensión-docencia-investigación. Finalmente, el Capítulo 11 ejerce una suerte de colofón que compara los vínculos de la extensión y la cultura en la Universidad Nacional de La Pampa.

Como puede apreciarse, los caminos de la extensión en la universidad pública argentina están siendo allanados. Si durante años la docencia y la investigación han gozado de una corporalidad física que las hizo protagonistas en los currículos universitarios, este libro es testigo de que las rutinas de trabajo y la cotidianeidad de la extensión son posibles... y necesarias. 